\title{
Mesoscopic organization of cobalt thin films on clean and oxygen-saturated $\mathrm{Fe}(001)$ surfaces
}

\author{
M. Riva, ${ }^{1}$ A. Picone,,${ }^{1,}$ D. Giannotti, ${ }^{1}$ A. Brambilla, ${ }^{1}$ G. Fratesi, ${ }^{2}$ G. Bussetti, ${ }^{1}$ L. Duò, ${ }^{1}$ F. Ciccacci, ${ }^{1}$ and M. Finazzi ${ }^{1}$ \\ ${ }^{1}$ Dipartimento di Fisica, Politecnico di Milano, Piazza Leonardo da Vinci 32, 20133 Milano, Italy \\ ${ }^{2}$ Dipartimento di Fisica, Università degli Studi di Milano, via Celoria 16, 20133 Milano, Italy
}

(Received 6 July 2015; published 21 September 2015)

\begin{abstract}
The different morphologies of Co films grown on either the clean $\mathrm{Fe}(001)$ surface and the oxygen-saturated $\mathrm{Fe}(001)-p(1 \times 1) \mathrm{O}$ substrate are investigated by means of scanning tunneling microscopy, Auger electron spectroscopy, and density functional theory. The considered Co coverage range extends beyond the thickness at which layer-by-layer growth is destabilized by plastic deformations induced by the relaxation of the strain accumulated in the film. Our findings indicate that the oxygen overlayer of the $\mathrm{Fe}(001)-p(1 \times 1) \mathrm{O}$ surface floats on top of the growing Co film and strongly influences both the Co nucleation process and the film structural evolution. The layer-dependent islands nucleation of Co films grown on clean $\mathrm{Fe}(001)$ substrates, recently associated with a thickness-dependent adatom mobility [A. Picone et al., Phys. Rev. Lett. 113, 046102 (2014)], is found to be suppressed by the oxygen overlayer. The latter also significantly delays the layer-by-layer instability with respect to the oxygen-free growth. Furthermore, the body-centered-tetragonal/hexagonal-close-packed transition is not observed in the case of $\mathrm{Co} / \mathrm{Fe}(001)-p(1 \times 1) \mathrm{O}$ sample, replaced by the development of highly ordered surface undulations. These form a mesoscopic square pattern with the sides aligned to the Fe $\langle 110\rangle$ directions, while the surface atomic structure retains the square $p(1 \times 1)$ symmetry in registry with the substrate. Such undulations are likely generated by a highly ordered array of interfacial misfit dislocations running along the $\mathrm{Fe}\langle 110\rangle$ directions.
\end{abstract}

DOI: 10.1103/PhysRevB.92.115434

PACS number(s): 68.37.Ef, 81.15.Aa, 68.55.J-

\section{INTRODUCTION}

The role played by an ordered overlayer of foreign atoms adsorbed on metals or semiconductor surfaces is a widely investigated topic in condensed matter physics and physical chemistry. Theoretical and experimental efforts have highlighted a rich phenomenology of adsorbate-induced effects, such as modifications of the work function [1,2] or surface electronic structure [3,4], enhanced [5-7] or tailored [8-14] surface magnetism, and structural relaxations [15-18], just to name a few. Aside from the above-mentioned phenomena, a widely investigated topic is the so-called surfactant-assisted epitaxial growth [19-24]. In this case, a monolayer (or less) of foreign atoms adsorbed on the substrate before film deposition strongly influences the film morphology, both for heteroepitaxy and homoepitaxy. The surfactant action has been demonstrated to be effective in the following:

(1) Inducing a surface flattening of the growing film [2528]. This phenomenon has been interpreted either in terms of a surfactant-induced reduction of the difference between the free-energy densities of the film and the substrate [19] or alteration of adatoms diffusion coefficients [20].

(2) Improving heteroepitaxial interfaces, reducing their roughness [29,30] and enhancing their chemical stability [30,31].

(3) Allowing the growth of thin oxide films characterized by a polar termination, otherwise unstable because of the presence of a diverging electrostatic dipole [32,33].

(4) Extending the stability regime of epitaxy-stabilized metastable structures [34-36].

In this paper, we compare the morphology obtained for ultrathin Co films deposited on the clean Fe(001) and on the oxygen-saturated $\mathrm{Fe}(001)-p(1 \times 1) \mathrm{O}$ surfaces. Previous

\footnotetext{
*andrea.picone@polimi.it
}

measurements performed with electron-based diffraction and spectroscopic techniques showed that $\mathrm{Co}$, characterized in its bulk form by hexagonal close-packed (hcp) crystal structure at room temperature, can be stabilized in a strained bodycentered tetragonal (bct) phase on top of the Fe(001) surface, up to a thickness of about $15 \AA[34,37,38]$. At larger thickness, a structural transition takes place, turning the metastable bct structure into the thermodynamically stable hcp phase, as evidenced by the development of a $c(2 \times 2)$ low-electronenergy diffraction (LEED) pattern [34].

Furthermore, it was reported that the presence of oxygen on the $\mathrm{Fe}(001)$ surface prior to Co deposition can extend the regime of bct stability up to about $30 \AA[34,35]$. Thicker Co films no longer produced measurable LEED patterns, therefore, the onset of the bct/hcp transition could not be observed, and the mechanisms behind the transition from the ordered to the disordered phase remained unclear. These phenomena are addressed in this paper, where we provide an atomic-scale view of the oxygen surfactant action during the growth of $\mathrm{Co}$ thin films on $\mathrm{Fe}(001)$ by means of scanning tunneling microscopy (STM).

We adopt the method discussed in Ref. [39] to precisely correlate the STM images with the absolute Co coverage by using wedged samples. This allowed us to directly compare the film morphologies obtained for Co thin films grown on the $\mathrm{Fe}(001)$ and on the oxygen-passivated $\mathrm{Fe}(001)-p(1 \times 1) \mathrm{O}$ surface, starting from submonolayer Co films up to the onset of the respective structural transitions. Our measurements reveal that oxygen significantly affects both the nucleation process and the mesoscopic morphological modifications occurring as the thickness approaches the limit at which the bct phase becomes unstable. The evolution of the film's morphology as a function of thickness is interpreted in terms of the energy associated with different atomic distributions at the film surface, as evaluated by calculations based on density functional theory (DFT). 


\section{EXPERIMENT}

Samples were prepared in an ultrahigh vacuum (UHV) system (low $10^{-10}$ mbar pressure range) by starting from a UHV-cleaned $\mathrm{MgO}(001)$ single-crystal substrate, over which a 400-nm-thick Fe(001) film was grown by means of molecular beam epitaxy $(\mathrm{MBE}) . \mathrm{Fe}(001)-p(1 \times 1) \mathrm{O}$ surfaces were obtained by exposing a clean $\mathrm{Fe}(001)$ substrate held at $500{ }^{\circ} \mathrm{C}$ to $30 \mathrm{~L}\left(1 \mathrm{~L}=1.3 \times 10^{-6}\right.$ mbar $\left.\times \mathrm{s}\right)$ of pure $\mathrm{O}_{2}$ (partial pressure: $\left.2.0 \times 10^{-7} \mathrm{mbar}\right)$. The samples were then heated at $700{ }^{\circ} \mathrm{C}$ for $10 \mathrm{~min}$ to remove the excess oxygen from the surface. This procedure generates an oxygen-saturated and well-ordered $\mathrm{Fe}(001)-p(1 \times 1) \mathrm{O}$ superstructure, characterized by one oxygen atom per surface unit cell, lying in the fourfold hollow site of the Fe surface lattice [3].

Co films were grown onto $\mathrm{Fe}(001)$ and $\mathrm{Fe}(001)-p(1 \times 1) \mathrm{O}$ substrates by MBE under UHV conditions, with a typical growth rate of about 0.12 equivalent monolayers (ML) per minute, ${ }^{1}$ as measured by a quartz microbalance. Wedged films, with Co thickness varying from 0 to $25 \mathrm{ML}$ for $\mathrm{Co} / \mathrm{Fe}(001)$ samples and from 0 to $47 \mathrm{ML}$ for $\mathrm{Co} / \mathrm{Fe}(001)-p(1 \times 1) \mathrm{O}$ samples, were obtained by shadowing the substrate with a movable shutter that was retracted at a constant rate during the growth, as discussed in Ref. [39].

Unless stated otherwise, the substrates were held at room temperature during Co deposition. The sample temperature was measured by a thermocouple mounted in close proximity of the sample position. STM images were acquired by using an Omicron Variable Temperature STM in a UHV chamber connected with the preparation system. STM measurements were acquired at room temperature in constant-current mode with home-made electrochemically etched W tips. After each acquisition of a STM image, the scan area was changed by means of the coarse motion of the piezoelectric drive, following the wedge from highest to lowest coverage. LEED and Auger electron spectroscopy (AES) data were acquired by means of an Omicron SPECTALEED with a retarding field analyzer (total acceptance angle $102^{\circ}$ ). A 3-kV, 20- $\mu \mathrm{A}$ electron beam was used, with a $3-\mathrm{V}$ peak-to-peak modulation amplitude. A selection of spectra is shown in Fig. 1, which indicates that the intensity of the oxygen-related peaks is unaffected by the presence of the Co film [see spectra (iii) and (iv)], thus indicating that oxygen keeps floating on top of the growing film.

\section{DFT SIMULATIONS}

DFT simulations were performed with ultrasoft pseudopotentials and plane waves as implemented in the QUANTUM ESPRESSO package [40] with the Perdew-Burke-Ernzerhof generalized gradient approximation [41] to the exchange and correlation functional. For $\mathrm{Co} / \mathrm{Fe}(001)-p(1 \times 1) \mathrm{O}$, pseudomorphic Co films were placed on top of a four-layer $\mathrm{Fe}(001)$

\footnotetext{
${ }^{1}$ One equivalent monolayer (1 ML) equals the amount of Co atoms required to completely saturate the adsorption sites on the Fe substrate, i.e., about $12.2 \times 10^{14}$ atoms $/ \mathrm{cm}^{2}$. The vertical height of $1 \mathrm{ML}$ is $1.433 \AA$, corresponding to half of a unit cell of body-centered-cubic Fe.
}

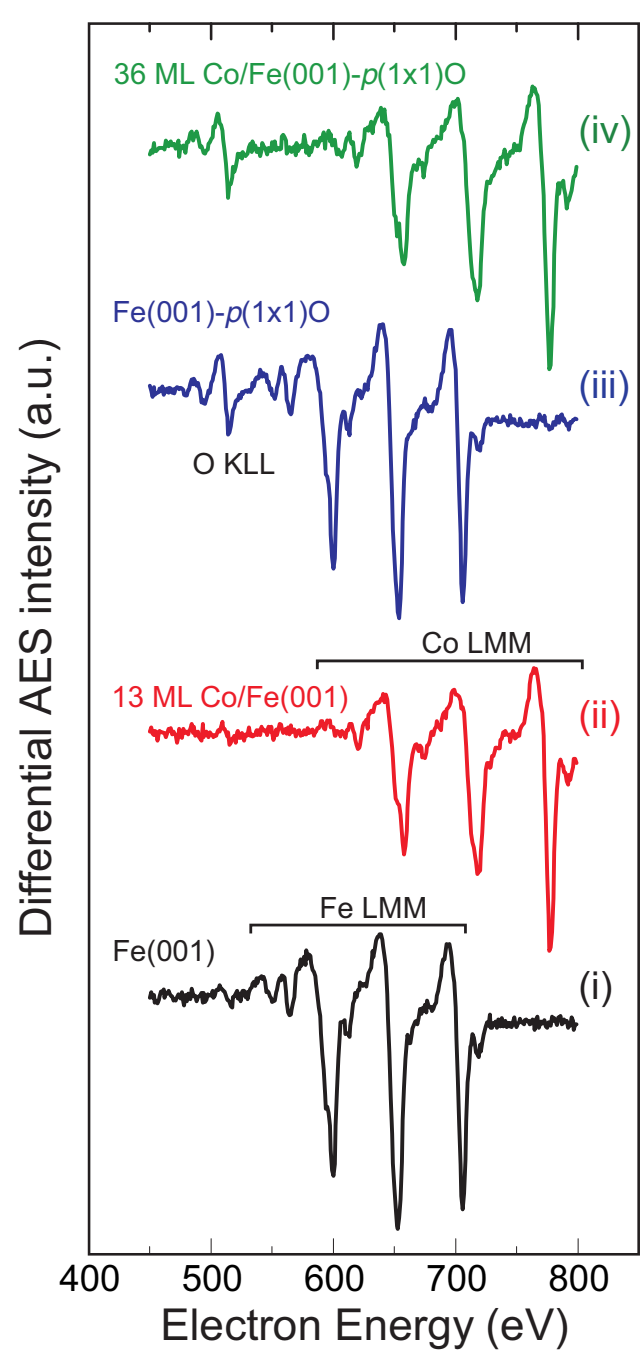

FIG. 1. (Color online) Auger electron spectroscopy data. Spectra (i) and (ii) were acquired before and after deposition of a 13-MLthick film of $\mathrm{Co}$ on the clean $\mathrm{Fe}(001)$ surface, respectively. Spectra (iii) and (iv) correspond to the oxygen-saturated $\mathrm{Fe}(001)-p(1 \times 1) \mathrm{O}$ surface and to a 36-ML-thick cobalt film grown on it, respectively. The intensity of the oxygen-related peak remains constant after $\mathrm{Co}$ deposition on the $\mathrm{Fe}(001)-p(1 \times 1) \mathrm{O}$, indicating that the oxygen layer keeps floating on top of the growing film.

slab, optimizing all atomic coordinates except the ones of the bottom two Fe layers. In describing Co adatoms, $3 \times 3$ surface supercells were adopted with a $5 \times 5$ sampling of the surface Brillouin zone. In the direction orthogonal to the slab, the system is separated from its replicas by $11-\AA$-thick vacuum portions.

The adsorption and diffusion of Co adatoms above the Co surface layer were evaluated by placing the adatom in the highsymmetry sites of the underlying Co lattice and optimizing the geometry. The case of $\mathrm{Co}$ on nonoxidized Fe(001)was discussed in Ref. [39], the hollow site $(\mathrm{H})$ being favored over the bridge (B) and top $(\mathrm{T})$ ones, as determined by the respective formation energy $F$, defined as

$$
F=\frac{E_{\mathrm{Co} / \mathrm{subs}}^{\mathrm{tot}}-E_{\mathrm{subs}}^{\mathrm{tot}}-\mu_{\mathrm{Co}} N_{\mathrm{Co}}}{N_{\mathrm{Co}}} .
$$


TABLE I. DFT results for the adatom formation energy $F$ above 1 ML Co on $\mathrm{Fe}(001)-p(1 \times 1) \mathrm{O}$, calculated for all the geometries shown in Fig. 2. Values in eV.

\begin{tabular}{lccccc}
\hline \hline Bridge & Hollow & Top & Hollow' & Above O & Exchange \\
\hline 0.82 & 1.82 & 2.68 & 2.03 & 3.14 & 2.07 \\
\hline \hline
\end{tabular}

Here, $E_{\mathrm{Co} / \mathrm{subs}}^{\text {tot }}$ is the DFT total energy of the combined system, $E_{\text {subs }}^{\text {tot }}$ that of the substrate (oxidized Fe or $\mathrm{Co} / \mathrm{Fe}$ film without adatoms), $\mu_{\mathrm{Co}}$ the chemical potential of Co adatoms (the reference being the bulk metal), and $N_{\text {Co }}$ their number. Lower values indicate more stable configurations.

Since oxygen floats on top of the Co film (see Fig. 1), the formation energy of $\mathrm{Co}$ adatoms on $\mathrm{Co} / \mathrm{Fe}(001)-p(1 \times 1) \mathrm{O}$ is evaluated by assuming that $\mathrm{O}$ atoms only occupy the $\mathrm{H}$ surface sites, with no $\mathrm{O}$ at the $\mathrm{Co} / \mathrm{Fe}$ interface. The presence of oxygen atoms drastically changes the picture derived for the Co growth on oxygen-free $\mathrm{Fe}(001)$, as it can already be seen from the
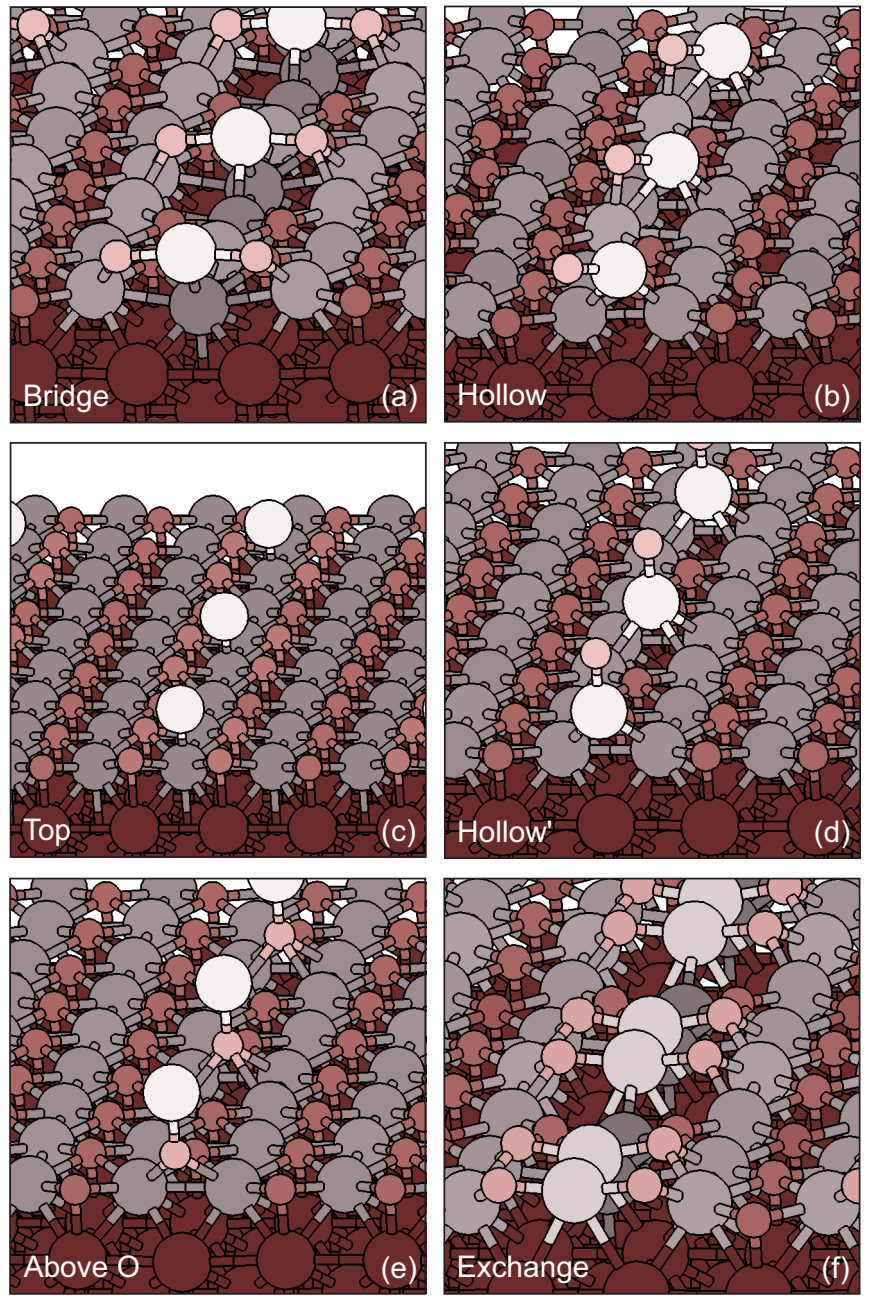

FIG. 2. (Color online) (a)-(e) Different adatom geometries considered for evaluating Co adsorption/diffusion on a 1-ML-thick Co film on $\mathrm{Fe}(001)-p(1 \times 1) \mathrm{O}$. (f) "Exchange" configuration where two Co atoms (the adatom plus an atom from the surface) lie at the same height above a Co-vacancy site. Small spheres represent $\mathrm{O}$ atoms. Different colors indicate atoms at different distances from the $\mathrm{Fe}$ layer.
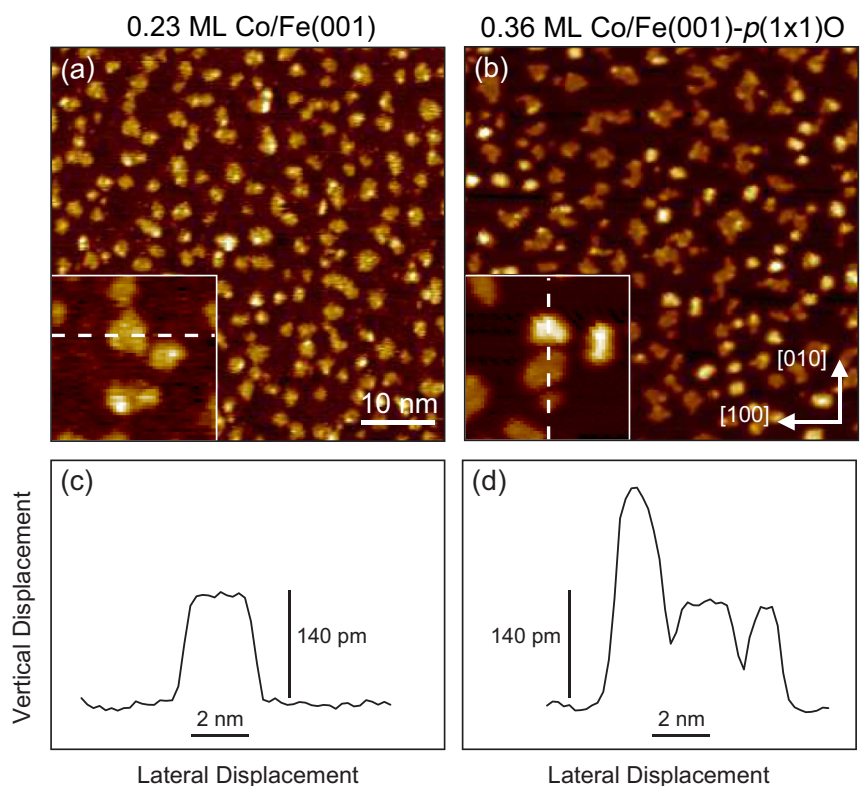

FIG. 3. (Color online) STM constant current topographies for submonolayer deposition of $\mathrm{Co}$ on (a) $\mathrm{Fe}(001)$ and on (b) the $\mathrm{Fe}(001)-p(1 \times 1) \mathrm{O}$ surface, respectively. Tunneling parameters for images in (a) and (b) are $V_{\mathrm{b}}=1.6 \mathrm{~V}, I_{\mathrm{t}}=1 \mathrm{nA}$ and $V_{\mathrm{b}}=1.5 \mathrm{~V}$, $I_{\mathrm{t}}=1 \mathrm{nA}$, respectively. (c), (d) Topographic height measured along the dashed lines of the insets inside panels (a) and (b), respectively.

$F$ values referring to Co adatoms on 1-ML-thick Co films on $\mathrm{Fe}(001)-p(1 \times 1) \mathrm{O}$ reported in Table I. We now find that, among the considered adatom geometries (see Fig. 2), the one characterized by the lowest $F$ value corresponds to Co atoms occupying B sites [Fig. 2(a)], being located between Co and $\mathrm{O}$ lattices, while Co occupying a $\mathrm{H}$ site [Fig. 2(b)] corresponds to a less favorable situation, $1 \mathrm{eV}$ higher in energy.

On the basis of the results in Table I, diffusion of Co adatoms can occur between two B sites by hopping through the $\mathrm{H}$ site. It is worth noticing that Co diffusion may proceed also through adatom exchange with a surface one. Such a diffusion path is characterized by small displacements in the $\mathrm{O}$ lattice. We evaluate the energetic cost of this exchange process by considering a symmetric "exchange" $(\mathrm{X})$ configuration where two Co atoms (the adatom plus an atom from the surface) lie at the same height above a Co-vacancy site [see Fig. 2(f)]. We obtain $F=2.07 \mathrm{eV}$, a value slightly larger but still comparable with that of a Co adatom occupying the $\mathrm{H}$ site. ${ }^{2}$

\section{RESULTS AND DISCUSSION}

\section{A. Submonolayer coverage}

Figure 3 compares the experimental morphology resulting from submonolayer deposition of $\mathrm{Co}$ on the bare $\mathrm{Fe}(001)$ and on the oxygen-saturated $\mathrm{Fe}(001)-p(1 \times 1) \mathrm{O}$ surface. In

\footnotetext{
${ }^{2}$ The exchange process was not discussed in Ref. [39] for Co diffusion on $\mathrm{Co} / \mathrm{Fe}(001)$. Actually, when there is no oxygen at the surface of the Co film, this mechanism is associated with a much higher barrier energy than the one characterizing H-B-H hopping $\left(F_{\mathrm{X}}-F_{\mathrm{H}}=1.46 \mathrm{eV}\right.$ vs $E_{b}=F_{\mathrm{B}}-F_{\mathrm{H}}=0.88 \mathrm{eV}$ for 1-ML-thick films).
} 
the former case, the Co coverage is about $0.23 \mathrm{ML}$, the island density being $8.93 \times 10^{-2} \mathrm{~nm}^{-2}$. In the latter, we measure a higher total coverage of $0.37 \mathrm{ML}$, yet with a lower island density of $6.05 \times 10^{-2} \mathrm{~nm}^{-2}$. Interestingly, while all islands covering the oxygen-free $\mathrm{Fe}(001)$ substrate are one atomic layer high, a non-negligible fraction of the islands growing on $\mathrm{Fe}(001)-p(1 \times 1) \mathrm{O}$ is two layers thick. For the sample reported in Fig. 3(b), about $30 \%$ and $3 \%$ of the substrate surface is covered by one-layer- and two-layer-thick islands, respectively. The tendency to form two-layer-thick islands was previously reported for $\mathrm{Ni}$ growth on $\mathrm{Fe}(001)$ $p(1 \times 1) \mathrm{O}$, probably related to a lower oxygen affinity of $\mathrm{Ni}$ with respect to the $\mathrm{Fe}$ substrate [42]. In that case, however, almost all the $\mathrm{Ni}$ islands growing on $\mathrm{Fe}(001)-p(1 \times 1) \mathrm{O}$ were two layers thick. DFT simulations provide a formation energy difference $F_{1 \mathrm{MLCo}}-F_{2 \mathrm{ML} \mathrm{Co}}=0.22 \mathrm{eV}$, indicating that two-layer-thick islands should be energetically favored with respect to monolayer ones. The stabilization is, however, somewhat smaller than for $\mathrm{Ni} / \mathrm{Fe}(001)-p(1 \times 1) \mathrm{O}$, for which $F_{1 \mathrm{MLNi}}-F_{2 \mathrm{ML} \mathrm{Ni}}=0.28 \mathrm{eV}$.

In order to verify that the thermodynamically most stable configuration corresponds to two-layer-thick islands, we also performed Co depositions at higher substrate temperatures (not shown). We find that increasing the temperature can indeed promote double-layer Co nucleation. For a 0.42-ML-thick Co film grown on the $\mathrm{Fe}(001)-p(1 \times 1) \mathrm{O}$ substrate held at $100^{\circ} \mathrm{C}$, we observe that the fraction of surface covered by two-layerthick islands is increased to $6 \%$, while $30 \%$ of the surface is covered by single-layer islands. At temperatures higher than $100{ }^{\circ} \mathrm{C}$ we observe Co diffusion into the substrate and the number of two-layer-thick islands drastically drops.

\section{B. Layer-by-layer growth and islands nucleation}

Figure 4 provides an overview of the growth behavior of the Co thin film on oxygen-free $\mathrm{Fe}(001)$ and on $\mathrm{Fe}(001)-$ $p(1 \times 1) \mathrm{O}$, as determined from the analysis of constant current STM images. As pointed out in our recent work [39], the Co

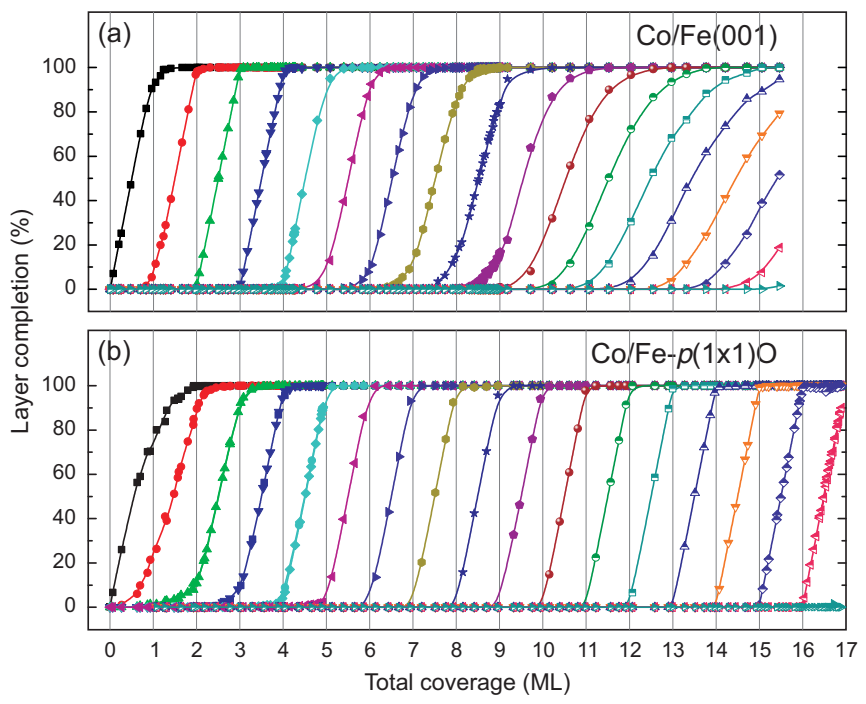

FIG. 4. (Color online) Layer completion as a function of the total Co coverage on (a) oxygen-free $\mathrm{Fe}(001)$ and on (b) $\mathrm{Fe}(001)$ $p(1 \times 1) \mathrm{O}$.
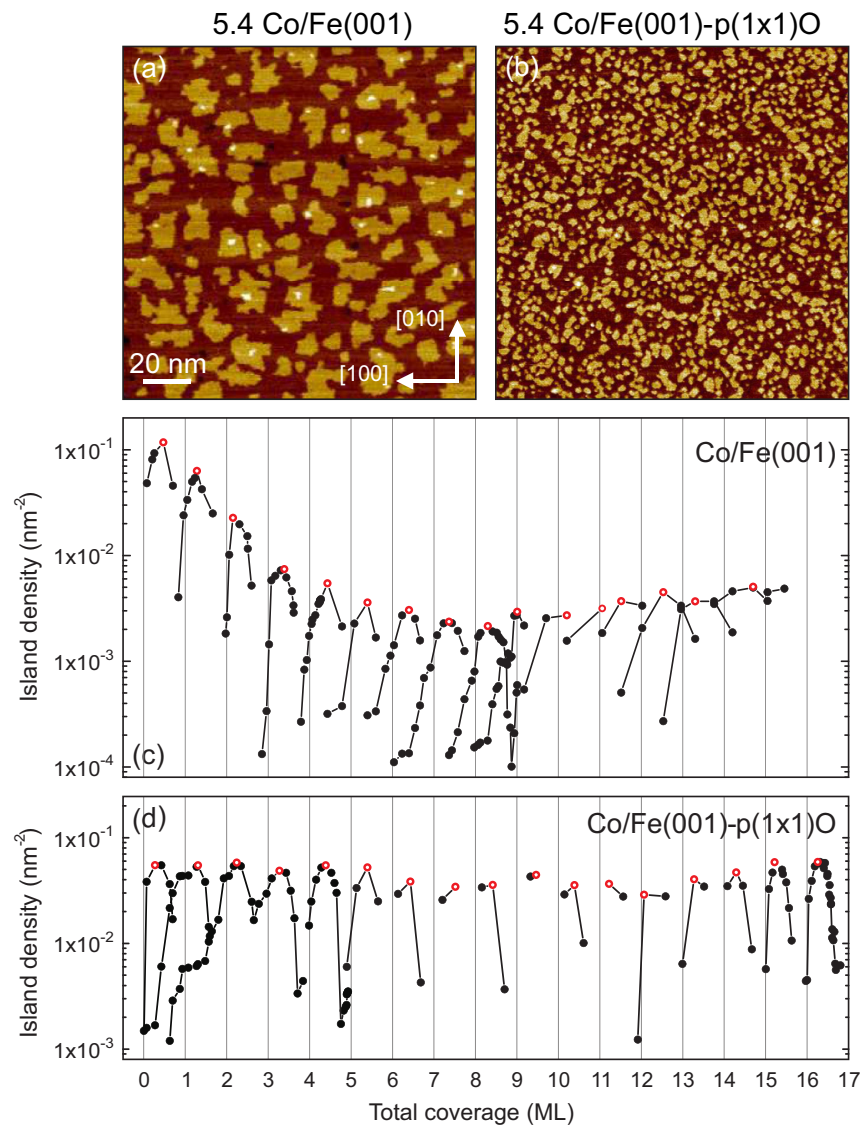

FIG. 5. (Color online) (a) STM topographic image of a 5.4-MLthick Co film grown on the oxygen-free $\mathrm{Fe}(001)$ surface. The island density is $3.60 \times 10^{-3} \mathrm{~nm}^{-2}$. (b) STM topographic image of a 5.4-ML-thick Co film grown on the oxygen-passivated $\mathrm{Fe}(001)$ $p(1 \times 1) \mathrm{O}$ surface. The island density is $5.25 \times 10^{-2} \mathrm{~nm}^{-2}$. (c), (d) Co island density as a function of the total coverage for Co films grown on (c) oxygen-free $\mathrm{Fe}(001)$ and on (d) $\mathrm{Fe}(001)-p(1 \times 1) \mathrm{O}$, respectively. The local maxima (hollow red dots) correspond to the saturation island density for a given layer.

growth on the oxygen-free surface proceeds in a layer-by-layer mode up to about $9 \mathrm{ML}$, showing a transition to multilayer growth for higher coverages. At a coverage of $15 \mathrm{ML}$, four atomic layers are partially completed, corresponding to five surface layers exposed. The Co growth mode on the $\mathrm{Fe}(001)-p(1 \times 1) \mathrm{O}$ surface is remarkably different. After a wetting layer is completed at a coverage equal to $2 \mathrm{ML}$, a nearly perfect layer-by-layer growth is initiated extending (up to $17 \mathrm{ML}$ ) considerably beyond the limit found for Co grown on the oxygen-free surface.

Figure 5 displays the layer-dependent saturation island density, defined as the maximum number of islands per unit area reached between two integer subsequent coverages. Striking differences can be observed for Co growth on the two different substrates. In the case of deposition onto oxygen-free $\mathrm{Fe}(001)$, the saturation island density follows a decreasing trend, starting from a value of $1.17 \times 10^{-1} \mathrm{~nm}^{-2}$ for the nucleated cobalt islands on the bare $\mathrm{Fe}(001)$ substrate, down to $2 \times 10^{-3} \mathrm{~nm}^{-2}$ for eight-layer islands (corresponding to Co adatom diffusion on the seventh layer). Conversely, when Co 
TABLE II. DFT results for the diffusion of Co adatoms over Co films deposited on $\mathrm{Fe}(001)-p(1 \times 1) \mathrm{O}\left(E_{\mathrm{HB}}=F_{\mathrm{H}}-F_{\mathrm{B}} ; E_{\mathrm{XB}}=\right.$ $\left.F_{\mathrm{X}}-F_{\mathrm{B}}\right)$ compared with the values of the diffusion barrier $E_{b}$ reported in Ref. [39] for $\mathrm{Co} / \mathrm{Fe}(001)$. Values in $\mathrm{eV}$.

\begin{tabular}{llcc}
\hline \hline & \multicolumn{2}{l}{$\mathrm{Co} / \mathrm{Fe}(001)-p(1 \times 1) \mathrm{O}$} & $\mathrm{Co} / \mathrm{Fe}(001)[39]$ \\
\cline { 2 - 3 } Coverage & $E_{\mathrm{HB}}$ & $E_{\mathrm{XB}}$ & $E_{b}$ \\
\hline $1 \mathrm{ML}$ & 1.00 & 1.25 & 0.88 \\
$2 \mathrm{ML}$ & 0.99 & 1.08 & 0.85 \\
$3 \mathrm{ML}$ & 1.11 & 0.93 & 0.55 \\
$4 \mathrm{ML}$ & 1.16 & 0.79 & 0.12 \\
$5 \mathrm{ML}$ & 1.14 & 0.71 & 0.00 \\
\hline \hline
\end{tabular}

is evaporated onto $\mathrm{Fe}(001)-p(1 \times 1) \mathrm{O}$, the saturation island density is not affected by the Co film thickness, keeping a constant value of about $5 \times 10^{-2} \mathrm{~nm}^{-2}$ across the $0-17 \mathrm{ML}$ coverage range.

In the framework of the classical nucleation theory, the island density is related to the diffusion rate of adatoms over the surface [43-45]. Accordingly, the different evolution of islands density in the two systems could be due to a different evolution of the respective diffusion coefficients. To better understand the trends shown in Figs. 5(c) and 5(d), we evaluated the B-H-B hopping barrier $E_{\mathrm{HB}}$ as the difference between the $\mathrm{H}$ and $\mathrm{B}$ formation energies calculated by DFT for a Co coverage ranging from 1 to $5 \mathrm{ML}$. Additionally, we estimated the activation barrier for Co-Co exchange $E_{\mathrm{XB}}$ by comparing the formation energies associated with the $\mathrm{X}$ and $\mathrm{B}$ geometries. The results are reported in Table II, which also displays the hopping barrier $E_{b}$ for the nonoxidized surface, from Ref. [39].

Two clearly different trends are obtained for the oxidized and oxygen-free substrates. In case of adatom diffusion on the surface of Co films on oxygen-free $\mathrm{Fe}(001)$, the value of $E_{b}$ decreases quickly as the coverage increases, a phenomenon that was associated with a softening of the Co film as the thickness approaches the limit at which the structural bct-hcp transition occurs [39]. Such a decrease in $E_{b}$ stems from the stabilization of adatom adsorption on the B site with respect to the $\mathrm{H}$ site: having a lower symmetry, the former is affected by larger structural deformations than the latter and is more favored by the thickness-dependent softening [39].

The different topology of the $\mathrm{Fe}(001)-p(1 \times 1) \mathrm{O}$ surface makes $\mathrm{B}$ a more stable configuration with respect to $\mathrm{H}$. Consequently, an opposite trend with respect to $\mathrm{Co} / \mathrm{Fe}(001)$ is observed for the hopping barrier $E_{\mathrm{HB}}$, which slowly increases with the number of Co layers. Actually, the situation is made even more complex by the presence of the additional diffusion channel involving the Co-Co exchange mechanism. The latter is less favorable than B-H-B hopping at low Co coverages, but becomes increasingly convenient as the Co film thickens. We can thus expect both processes to contribute to Co adatom diffusion, possibly with different relative weights as a function of the Co coverage. Eventually, DFT calculations seem to be in good agreement with the observed dramatically different trends shown in Figs. 5(c) and 5(d) since they predict a diffusion barrier for $\mathrm{Co} / \mathrm{Fe}(001)-p(1 \times 1) \mathrm{O}$ which does not decrease as rapidly with increasing Co coverage as for $\mathrm{Co} / \mathrm{Fe}(001)$.
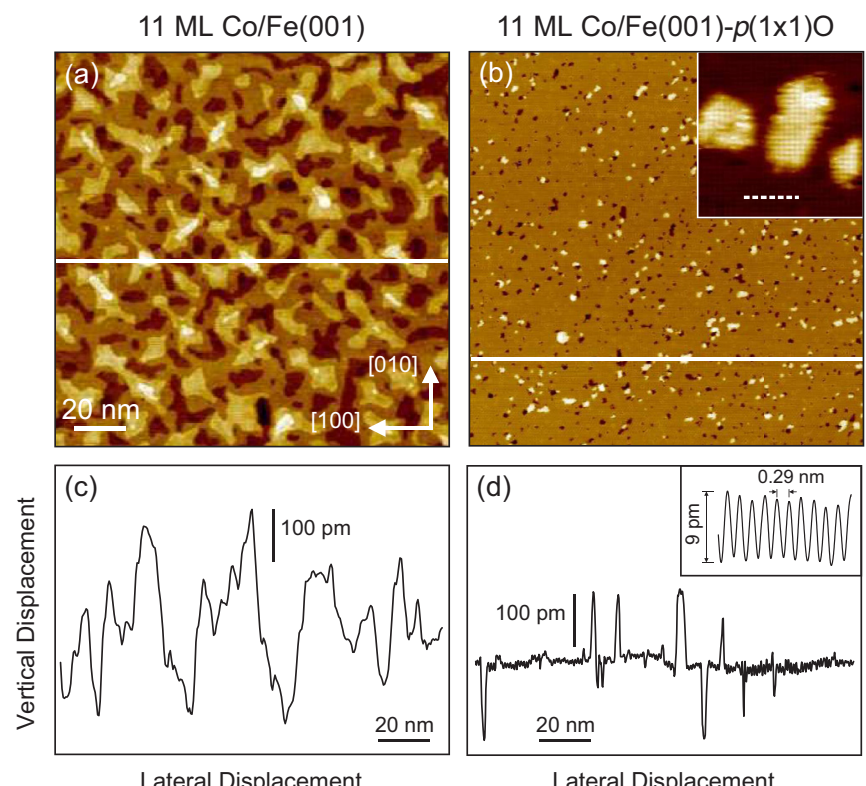

Lateral Displacement

FIG. 6. (Color online) Surface morphologies of $11 \mathrm{ML}$ of Co deposited on (a) the oxygen-free $\mathrm{Fe}(001)$ surface and on (b) the $\mathrm{Fe}(001)-p(1 \times 1) \mathrm{O}$ substrate, as evaluated from STM constant current images. The topographic heights corresponding to the continuous white lines in (a) and (b) are displayed in (c) and (d), respectively. The inset in panel (b) shows an atomically resolved STM image acquired in a region straddling a terrace and some Co islands. The measured atomic corrugation, corresponding to the dashed white line, is shown in the inset of panel (d). Tunneling parameters are $V_{\mathrm{b}}=1 \mathrm{~V}$, $I_{\mathrm{t}}=500 \mathrm{pA}$ for images in (a) and (b). Tunneling parameters for the atomically resolved image in the inset of panel (b) are $V_{\mathrm{b}}=0.1 \mathrm{~V}$, $I_{\mathrm{t}}=5 \mathrm{nA}$.

\section{Destabilization of the bct phase}

Figure 6 compares the surface morphology of 11-ML-thick Co films, a thickness that is slightly above the threshold of the bct to hcp transition for Co grown on oxygen-free Fe(001) [34]. At such thickness, Co films grown on the oxygen-free $\mathrm{Fe}(001)$ substrate feature a multilayer morphology, as testified by Fig. 6(c). In addition, it is possible to notice that Co atoms form mounds which are elongated along the $\langle 110\rangle$ in-plane crystallographic directions, similar to those observed in the martensitic transition of $\mathrm{Ni}$ films grown on $\mathrm{Fe}(001)$ [46,47]. On the other hand, at the same coverage, the topography of the Co film grown on the oxygen-saturated surface is atomically flat, with basically only one exposed layer, as shown in Fig. 6(d). Atomically resolved images acquired over a region straddling a terrace and an island [see inset of Fig. 6(b)] show that both the islands and the terrace are highly ordered and characterized by a square symmetry. In both cases, the atomic corrugation measured in constant current images is about $9 \mathrm{pm}$, with a periodicity along the [100] direction of approximately $2.9 \AA$ [see inset of Fig. 6(d)], nicely corresponding to the lattice constant of the $\mathrm{Fe}(001)$ surface $\left(a_{\mathrm{Fe}}=2.866 \AA\right)$. This measurement in particular confirms that the oxygen atoms arrange in a highly ordered and compact overlayer, completely saturating the surface.

Figure 7 focuses on the morphology of a 15-ML-thick Co film grown on the oxygen-free $\mathrm{Fe}(001)$ substrate, i.e., for 


\section{$15 \mathrm{ML} \mathrm{Co} / \mathrm{Fe}(001)$}
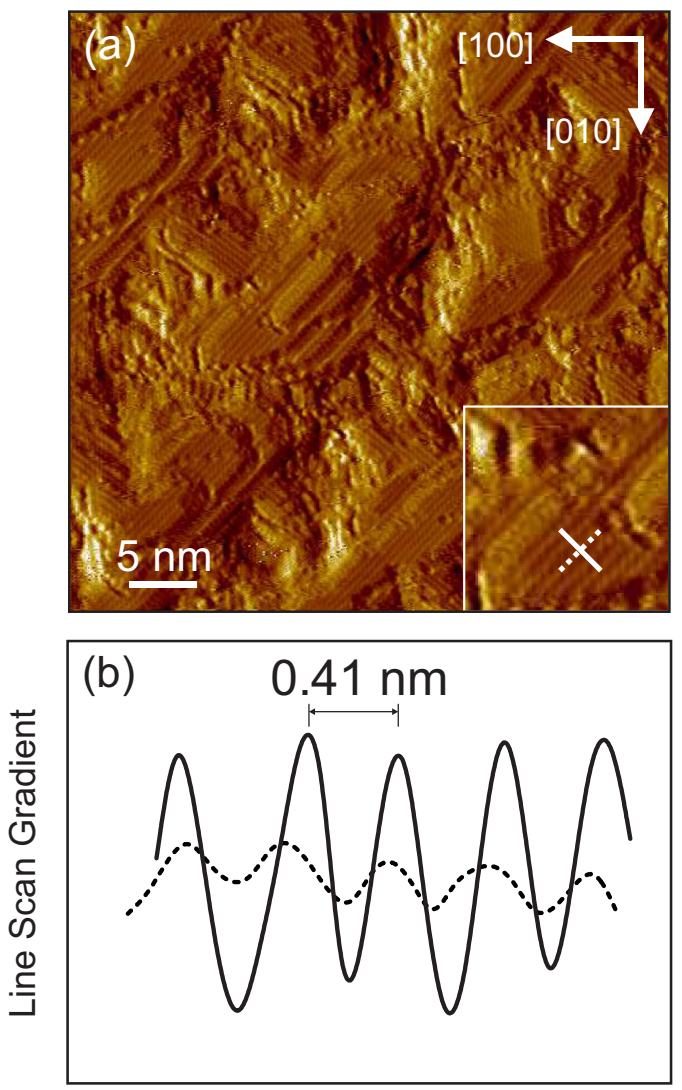

Lateral Displacement

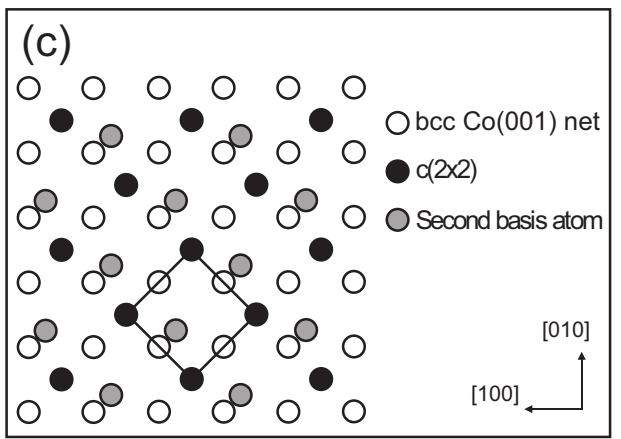

FIG. 7. (Color online) (a) Gradient of the STM topography acquired on a 15-ML-thick Co film grown on the oxygen-free $\mathrm{Fe}(001)$ substrate. Tunneling parameters are $V_{\mathrm{b}}=0.5 \mathrm{~V}, I_{\mathrm{t}}=400 \mathrm{pA}$. (b) Line scans corresponding to the continuous and dashed lines. The corrugation gradient measured along the [110] direction (dashed line) is weaker than that measured along the orthogonal direction (continuous line). (c) Model proposed for the topmost layer of hcp cobalt $[34,48]$. The compressed square net is rotated by $45^{\circ}$ with respect to the [001] substrate direction.

a coverage at which Kim et al. reported the presence of a well-developed $c(2 \times 2)$ LEED pattern [34]. In Fig. 7(a), we show the gradient of the constant current STM image in order to evidence the atomic-scale topographic structures, otherwise overwhelmed by the mesoscopic surface topography. Figure 7(b) reports the line scans corresponding to the continuous and dashed lines of the inset of Fig. 7(a). In both cases,
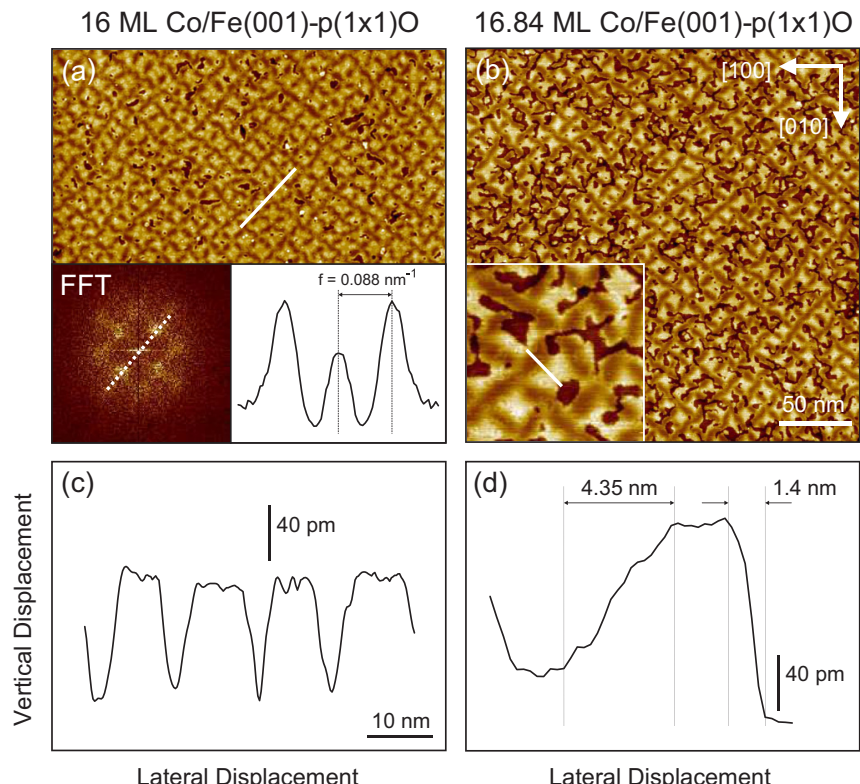

FIG. 8. (Color online) (a) Large-scale STM image of a 16-MLthick Co film grown on the $\mathrm{Fe}(001)-p(1 \times 1) \mathrm{O}$ surface. The inset on the left shows the FFT of the STM image, from which a periodicity of $11.36 \mathrm{~nm}$ can be measured (see in the inset on the right the profile traced along the dashed white line). (b) Large-scale STM image of a 16.84-ML-thick Co film grown on the $\mathrm{Fe}(001)-p(1 \times 1) \mathrm{O}$ surface, with an enlarged view displayed in the inset. (c), (d) STM profiles measured along the lines traced in (a) and (b), respectively. Tunneling parameters for the images in (a) and (b) are $V_{\mathrm{b}}=1 \mathrm{~V}, I_{\mathrm{t}}=1 \mathrm{nA}$ and $V_{\mathrm{b}}=1 \mathrm{~V}, I_{\mathrm{t}}=800 \mathrm{pA}$, respectively.

the corrugation periodicity is about $0.41 \mathrm{~nm}$, corresponding to the $\mathrm{Fe}(001)$ lattice periodicity along the [110] direction $\sqrt{2} \times a_{\mathrm{Fe}}=4.053 \AA$. Interestingly, the corrugation intensity measured along the [110] direction is different from that measured along the [1 10$]$ direction. Two orthogonal domains are visible in the image. To understand this STM image, we recall the model proposed by Kim et al. [34], who report the Co bct to hcp transition as inferred from the development of a $c(2 \times 2)$ LEED pattern. The latter was interpreted as due to hcp Co exposing the $(11 \overline{2} 0)$ face, with the $(11 \overline{2} 0)$ rectangular net compressed and rotated by $45^{\circ}$ with respect to the in-plane $\langle 001\rangle$ directions of the substrate [see Fig. 7(c)]. The basis of the compressed rectangular lattice is composed by two atoms. We suggest that the two orthogonal domains visible in Fig. 7(a) depend on the different disposition of the rectangular hcp (1120) net with respect to the square surface lattice, i.e., with the long side aligned along either the [110] or the [110] direction.

Figure 8 (a) displays the sample morphology of a 16-MLthick Co film grown on the $\mathrm{Fe}(001)-p(1 \times 1) \mathrm{O}$ surface, which is not atomically flat due to the presence of periodic surface undulations, arranged in a square pattern rotated by $45^{\circ}$ with respect to the $\langle 110\rangle$ directions of the substrate. ${ }^{3}$ This superstructure is characterized by a periodicity of about $11.36 \mathrm{~nm}$,

\footnotetext{
${ }^{3}$ The periodic surface undulations start to appear as faint features in STM images corresponding to 14-ML-thick Co films grown on $\mathrm{Fe}(001)-p(1 \times 1) \mathrm{O}$.
} 
as can be inferred from the fast Fourier transform (FFT) of the STM image [see inset of Fig. 8(a)]. Figure 8(c) displays a line scan corresponding to the sample of Fig. 8(a), showing that at this coverage the undulations are about $80 \mathrm{pm}$ high. Surface undulations are present also for fractional coverages, where monoatomic steps are also visible, as demonstrated by the topographic image shown in Fig. 8(b), corresponding to a Co coverage of 16.84 ML. Figure 8(d) shows a line scan acquired in a region straddling a surface undulation and an atomic step. This image allows one to directly compare the slope measured across a monoatomic Co step and across the undulation. The sharpness of the line scan across the monoatomic step is limited by tip/step convolution effects, resulting in a measured lateral width of $1.4 \mathrm{~nm}$. Conversely, the profile slope is less steep across the surface undulation, with a lateral width of $4.35 \mathrm{~nm}$.

A different mesoscopic pattern, still arranged according with a square lattice rotated by $45^{\circ}$ with respect to the $\langle 110\rangle$ directions of the substrate, is visible in the surface topography of a 18-ML-thick Co film grown on $\mathrm{Fe}(001)-p(1 \times 1) \mathrm{O}$ [see Fig. 9(a)]. Within the experimental uncertainty, the superlattice parameter is the same as the one measured for the 16-MLthick film. In the inset of Fig. 9(a), a blowup is presented, where atomic resolution allows one to detect a square lattice in registry with the substrate, superposed on a conspicuous continuous surface distortion. The topographical elevation of such a distortion appears to be larger than the one observed on the 16-ML-thick sample, as testified by the line scan reaching a corrugation of about 200 pm, as shown in Fig. 9(b). At a Co coverage higher than $18 \mathrm{ML}$, the roughness associated with the surface distortion further increases, in agreement with the disordered phase reported in Ref. [34]. However, we were able to acquire atomically resolved images (not shown) also in these samples, still observing a square lattice.

In order to explain the development of the mesoscopic square superlattice observed in Co films grown on $\mathrm{Fe}(001)$ $p(1 \times 1) \mathrm{O}$ from about $14 \mathrm{ML}$ on, we recall that wavelike surface modulations have been related to the development of buried misfit dislocations [49-52]. The dislocation network is often observed to develop at correspondence with the coincidence-site lattice (CSL) between the overlayer and the substrate [53,54]. The CSL is defined as the smallest lattice which is common to the two primitive ones [53,55]. Apparently, the dislocation lines in $\mathrm{Co} / \mathrm{Fe}(001)-p(1 \times 1) \mathrm{O}$ remain entirely confined within the film. A possible explanation could be that intercepting the surface would be energetically unfavorable because the strong O-Co covalent bonds would have to be broken and rearranged.

Figure 9(c) displays a geometrical model that can be applied for the interpretation of the surface deformation network in terms of misfit dislocations. The diagram represents the superposition of two square lattices (black continuous lines and red dashed lines) where the lattice misfit is intentionally exaggerated to highlight the coincidence points. Depending on the position of the overlayer lattice sites with respect to the substrate lattice, different coincidence lattices can be formed. In Fig. 9(c), the coincidence points corresponding to top, bridge, and hollow epitaxial sites are marked with circles, triangles, and squares, respectively. The CSL corresponding to top and hollow sites are aligned with the two primitive lattices. Their periodicity $\Lambda$ is given by $\Lambda=a_{0} /|f|$, with
$18 \mathrm{ML} \mathrm{Co} / \mathrm{Fe}(001)-p(1 \times 1) \mathrm{O}$
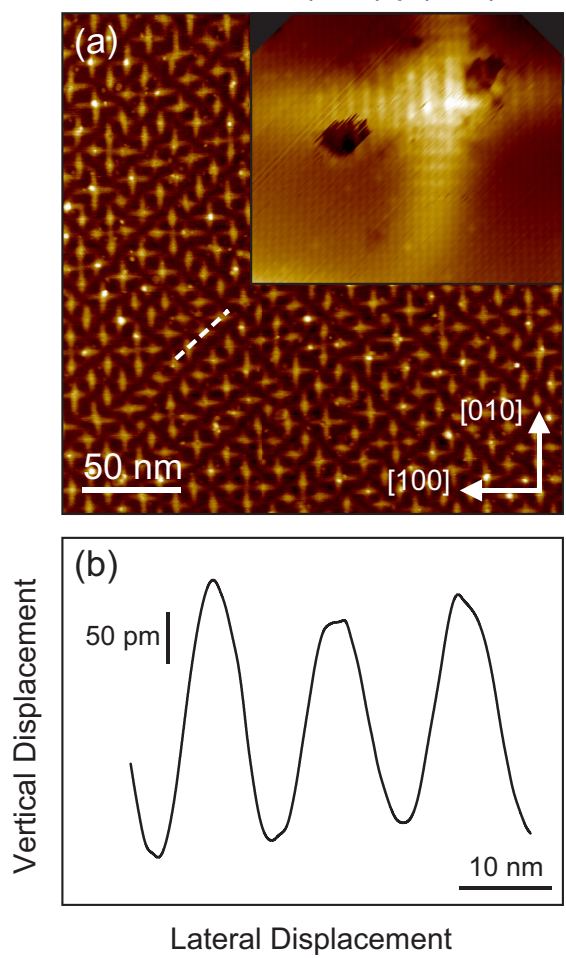

(c)

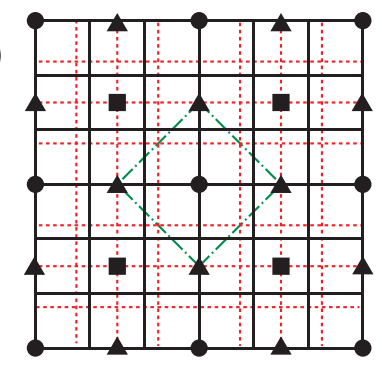

FIG. 9. (Color online) (a) Large-scale STM image of an 18-MLthick Co film grown on the $\mathrm{Fe}(001)-p(1 \times 1) \mathrm{O}$ surface. Inset: atomically resolved image of the distorted surface. (b) STM profile measured along the line traced in (a). Tunneling parameters are $V_{\mathrm{b}}=1 \mathrm{~V}, I_{\mathrm{t}}=500 \mathrm{pA}$. (c) Superposition of two square lattices (continuous black lines and dashed red lines) with a lattice mismatch $|f|=0.75$. The coincidence points corresponding to epilayer atoms placed on the hollow, top, and bridge epitaxial sites are shown as squares, circles, and triangles, respectively. The dashed-dotted green line represents the unit cell of the CSL corresponding to bridge epitaxial sites.

$f=\left(a_{\mathrm{o}}-a_{\mathrm{s}}\right) / a_{\mathrm{s}}$, where $a_{\mathrm{o}}$ and $a_{\mathrm{s}}$ are the lattice constants of the overlayer and the substrate, respectively. Indeed, on the other hand, the CSL corresponding to the bridge epitaxial sites is rotated by $45^{\circ}$ with respect to the primitive lattices, with a lattice parameter smaller by a factor $\sqrt{2}$. Since our experimental results (Figs. 8 and 9) show that the mesoscopic square network is rotated by $45^{\circ}$ with respect to the square net of the substrate, the coincidence lattice to be considered is that formed by the bridge sites. Moreover, the measured periodicity along the [110] direction $(11.36 \mathrm{~nm})$ is in good agreement with the CSL that would be obtained by considering the bridge coincidence points. Indeed, the hypothetical bulk phase of 
body-centered-cubic (bcc) Co would possess a lattice constant $a_{\mathrm{Co}_{\mathrm{bcc}}}=2.819 \AA$ [56], yielding a mismatch $|f|=0.016$ with respect to the $\mathrm{Fe}(001)$ lattice $\left(a_{\mathrm{Fe}}=2.866 \AA\right)$, leading to $\Lambda=a_{\mathrm{Co}_{\mathrm{bcc}}} /(\sqrt{2} \times|f|)=12.45 \mathrm{~nm}$.

Different crystal structures for the Co films are unlikely. A possible film transition involving the development of facecentered-cubic (fcc) phase (squared primitive unit mesh with a lattice constant $a_{\mathrm{Co}_{\text {fcc }}}=2.506 \AA$ ) [57] would lead to a superstructure periodicity $\Lambda=a_{\mathrm{Co}_{\mathrm{fcc}}} /\left(\sqrt{2} \times\left|f_{\mathrm{fcc}}\right|\right)=1.99 \mathrm{~nm}$, by far too small with respect to the one we measure. On the other hand, a transition to the hcp film structure would instead produce CLS without square symmetry since the matching of the $\operatorname{Co}(11 \overline{2} 0)$ rectangular net onto the bcc $\mathrm{Fe}(001)$ lattice requires $6.64 \%$ compression on the long side and a $0.40 \%$ compression on the short side. We thus suggest that Co films deposited onto $\mathrm{Fe}(001)-p(1 \times 1) \mathrm{O}$ remain in the bct phase for a layer thickness up to $18 \mathrm{ML}$ and that tensile strain is then relaxed by the insertion of edge-type dislocations running along the $\mathrm{Fe}\langle 110\rangle$ in-plane directions.

\section{CONCLUSIONS}

Our data show that the presence of a single layer of oxygen at the surface of Co films growing on $\mathrm{Fe}(001)-p(1 \times 1) \mathrm{O}$ substrates drastically affects both the island nucleation process and the mesoscopic surface morphology as the film thickness is increased from submonolayer coverage up to the limit at which the bct phase becomes unstable.

First, oxygen tends to favor the nucleation of two-layerthick Co islands before a wetting layer is completed. Second, in the 2-9 ML coverage range, the growth proceeds in an almost perfect layer-by-layer mode for films grown on both the oxygen-free $\mathrm{Fe}(001)$ and on the $\mathrm{Fe}(001)-p(1 \times 1) \mathrm{O}$ substrates. However, the layer-dependent island nucleation, characteristic of the $\mathrm{Co} / \mathrm{Fe}(001)$ system [39], is found to be suppressed by the presence of the oxygen overlayer. Third, at coverages above $9 \mathrm{ML}$, the well-known bct/hcp structural transition occurring for Co grown on oxygen-free $\mathrm{Fe}(001)$ is accompanied by a surface morphological evolution, characterized by the development of mesoscopic mounds elongated along the in-plane $\langle 110\rangle$ crystallographic substrate directions. Conversely, Co films grown on the $\mathrm{Fe}(001)-p(1 \times 1) \mathrm{O}$ surface are atomically flat up to $14 \mathrm{ML}$, displaying a square symmetry. The surface of Co films thicker than $14 \mathrm{ML}$ is characterized by the development of a pattern of highly ordered undulations, most likely generated by the presence of a square dislocation network, with dislocation lines running along the in-plane $\mathrm{Fe}\langle 110\rangle$ directions.

We suggest that the atomically flat bct Co films stabilized on the $\mathrm{Fe}(001)-p(1 \times 1) \mathrm{O}$ surface could be an ideal substrate for the growth of high-quality rocksalt structured mono-oxides [58-61], while thicker films could represent an interesting benchmark for the study of the strain relaxation mechanisms driving the destabilization of the Co metastable bct phase [62].

\section{ACKNOWLEDGMENTS}

We acknowledge the CINECA Award No. HP10CESYLM, 2014, for the availability of high performance computing resources and support. This work was partially supported by the Italian Ministry of University and Research through the FIRB Project No. RBAP115AYN.
[1] H. W. Hugosson, W. Cao, S. Seetharaman, and A. Delin, J. Phys. Chem. C 117, 6161 (2013).

[2] A. Michaelides, P. Hu, M.-H. Lee, A. Alavi, and D. A. King, Phys. Rev. Lett. 90, 246103 (2003).

[3] F. Donati, P. Sessi, S. Achilli, A. Li Bassi, M. Passoni, C. S. Casari, C. E. Bottani, A. Brambilla, A. Picone, M. Finazzi, L. Duò, M. I. Trioni, and F. Ciccacci, Phys. Rev. B 79, 195430 (2009).

[4] A. Picone, G. Fratesi, A. Brambilla, P. Sessi, F. Donati, S. Achilli, L. Maini, M. I. Trioni, C. S. Casari, M. Passoni, A. Li Bassi, M. Finazzi, L. Duò, and F. Ciccacci, Phys. Rev. B 81, 115450 (2010).

[5] A. Tange, C. Gao, W. Wulfhekel, and J. Kirschner, Phys. Rev. B 81, 220404 (2010).

[6] R. Bertacco and F. Ciccacci, Phys. Rev. B 59, 4207 (1999).

[7] A. Tange, C. L. Gao, B. Y. Yavorsky, I. V. Maznichenko, C. Etz, A. Ernst, W. Hergert, I. Mertig, W. Wulfhekel, and J. Kirschner, Phys. Rev. B 81, 195410 (2010).

[8] F. Bisio, R. Moroni, M. Canepa, L. Mattera, R. Bertacco, and F. Ciccacci, Phys. Rev. Lett. 83, 4868 (1999).

[9] J. Hong, R. Q. Wu, J. Lindner, E. Kosubek, and K. Baberschke, Phys. Rev. Lett. 92, 147202 (2004).

[10] D. Klar, B. Brena, H. C. Herper, S. Bhandary, C. Weis, B. Krumme, C. Schmitz-Antoniak, B. Sanyal, O. Eriksson, and H. Wende, Phys. Rev. B 88, 224424 (2013).
[11] F. Máca, J. Kudrnovský, V. Drchal, and J. Redinger, Phys. Rev. B 88, 045423 (2013).

[12] W. Feng, H. L. Meyerheim, K. Mohseni, O. Brovko, V. S. Stepanyuk, N. Jedrecy, R. Felici, and J. Kirschner, Phys. Rev. Lett. 110, 235503 (2013).

[13] C. Thiede, C. Langenkämper, K. Shirai, A. B. Schmidt, T. Okuda, and M. Donath, Phys. Rev. Applied 1, 054003 (2014).

[14] G. Berti, A. Brambilla, A. Calloni, G. Bussetti, M. Finazzi, L. Duò, and F. Ciccacci, Appl. Phys. Lett. 106, 162408 (2015).

[15] S. R. Chubb and W. E. Pickett, Phys. Rev. Lett. 58, 1248 (1987).

[16] A. Eichler and J. Hafner, Phys. Rev. B 62, 5163 (2000).

[17] S. S. Parihar, H. L. Meyerheim, K. Mohseni, S. Ostanin, A. Ernst, N. Jedrecy, R. Felici, and J. Kirschner, Phys. Rev. B 81, 075428 (2010).

[18] V. C. Schwindt, J. Ardenghi, P. Bechthold, E. Gonzàlez, P. Jasen, A. Juan, B. Batic, and M. Jenko, Appl. Surf. Sci. 315, 252 (2014)

[19] M. Copel, M. C. Reuter, E. Kaxiras, and R. M. Tromp, Phys. Rev. Lett. 63, 632 (1989).

[20] J. Camarero, J. Ferrón, V. Cros, L. Gómez, A. L. Vázquez de Parga, J. M. Gallego, J. E. Prieto, J. J. de Miguel, and R. Miranda, Phys. Rev. Lett. 81, 850 (1998).

[21] H. L. Meyerheim, D. Sander, R. Popescu, W. Pan, I. Popa, and J. Kirschner, Phys. Rev. Lett. 99, 116101 (2007).

[22] K.-J. Hsueh, C.-J. Tsai, S.-Y. Wu, H.-L. Chou, F. Bisio, C.-C. Kuo, and W.-C. Lin, J. Appl. Phys. 114, 203907 (2013). 
[23] C.-J. Tsai, K.-J. Hsueh, N. Plusnin, C.-C. Kuo, and W.-C. Lin, Appl. Surf. Sci. 313, 166 (2014).

[24] Z.-j. Wang, A. Dong, M. Wei, Q. Fu, and X. Bao, Appl. Phys. Lett. 104, 181604 (2014).

[25] A. Picone, A. Brambilla, A. Calloni, L. Duò, M. Finazzi, and F. Ciccacci, Phys. Rev. B 83, 235402 (2011).

[26] A. Calloni, A. Picone, A. Brambilla, M. Finazzi, L. Duò, and F. Ciccacci, Surf. Sci. 605, 2092 (2011).

[27] H. Fukumoto, M. Miyazaki, Y. Aoki, K. Nakatsuji, and H. Hirayama, Surf. Sci. 611, 49 (2013).

[28] T. Kojima, M. Mizuguchi, and K. Takanashi, Surf. Sci. 619, 44 (2014).

[29] S. Amir, M. Gupta, A. Gupta, and J. Stahn, Appl. Phys. A 111, 495 (2013).

[30] S. M. Amir, M. Gupta, S. Potdar, A. Gupta, and J. Stahn, J. Appl. Phys. 114, 024307 (2013).

[31] A. Tekiel, J. Topple, Y. Miyahara, and P. Grütter, Nanotechnology 23, 505602 (2012).

[32] X. Tan and P. Zapol, Phys. Rev. B 86, 045422 (2012).

[33] V. K. Lazarov, Z. Cai, K. Yoshida, K. H. L. Zhang, M. Weinert, K. S. Ziemer, and P. J. Hasnip, Phys. Rev. Lett. 107, 056101 (2011).

[34] S. K. Kim, C. Petersen, F. Jona, and P. M. Marcus, Phys. Rev. B 54, 2184 (1996).

[35] L. Duò, R. Bertacco, G. Isella, F. Ciccacci, and M. Richter, Phys. Rev. B 61, 15294 (2000).

[36] M. Á. Niño, J. Camarero, L. Gómez, J. Ferrón, J. J. de Miguel, and R. Miranda, J. Phys.: Condens. Matter 20, 265008 (2008).

[37] G. C. Gazzadi and S. Valeri, Europhys. Lett. 45, 501 (1999).

[38] H. Li and B. P. Tonner, Phys. Rev. B 40, 10241 (1989).

[39] A. Picone, M. Riva, G. Fratesi, A. Brambilla, G. Bussetti, M. Finazzi, L. Duò, and F. Ciccacci, Phys. Rev. Lett. 113, 046102 (2014).

[40] P. Giannozzi, S. Baroni, N. Bonini, M. Calandra, R. Car, C. Cavazzoni, D. Ceresoli, G. L. Chiarotti, M. Cococcioni, I. Dabo, A. D. Corso, S. de Gironcoli, S. Fabris, G. Fratesi, R. Gebauer, U. Gerstmann, C. Gougoussis, A. Kokalj, M. Lazzeri, L. Martin-Samos, N. Marzari, F. Mauri, R. Mazzarello, S. Paolini, A. Pasquarello, L. Paulatto, C. Sbraccia, S. Scandolo, G. Sclauzero, A. P. Seitsonen, A. Smogunov, P. Umari, and R. M. Wentzcovitch, J. Phys.: Condens. Matter 21, 395502 (2009).

[41] J. P. Perdew, K. Burke, and M. Ernzerhof, Phys. Rev. Lett. 77, 3865 (1996).
[42] A. Picone, G. Bussetti, M. Riva, A. Calloni, A. Brambilla, L. Duò, F. Ciccacci, and M. Finazzi, Phys. Rev. B 86, 075465 (2012).

[43] H. Brune, Surf. Sci. Rep. 31, 125 (1998).

[44] Islands Mounds and Atoms, edited by T. Michely and J. Krug (Springer, Berlin, 2004).

[45] M. Einax, W. Dieterich, and P. Maass, Rev. Mod. Phys. 85, 921 (2013).

[46] G. Bussetti, M. Riva, A. Picone, A. Brambilla, L. Duò, M. Finazzi, and F. Ciccacci, New J. Phys. 14, 053048 (2012).

[47] G. Bussetti, M. Riva, A. Picone, A. Brambilla, L. Duò, F. Ciccacci, and M. Finazzi, Nanosci. Nanotechnol. Lett. 4, 1092 (2012).

[48] C. P. Wang, S. C. Wu, F. Jona, and P. M. Marcus, Phys. Rev. B 49, 17391 (1994).

[49] R. Stalder, H. Sirringhaus, N. Onda, and H. von Känel, Appl. Phys. Lett. 59, 1960 (1991).

[50] G. Springholz, Appl. Surf. Sci. 112, 12 (1997).

[51] G. Jnawali, H. Hattab, F.-J. Meyer zu Heringdorf, B. Krenzer, and M. Horn-von Hoegen, Phys. Rev. B 76, 035337 (2007).

[52] G. Jnawali, H. Hattab, C. Bobisch, A. Bernhart, E. Zubkov, R. Möller, and M. H. von Hoegen, Surf. Sci. 603, 2057 (2009).

[53] G. Renaud, P. Guénard, and A. Barbier, Phys. Rev. B 58, 7310 (1998).

[54] P. Torelli, E. Soares, G. Renaud, S. Valeri, X. Guo, and P. Luches, Surf. Sci. 601, 2651 (2007).

[55] W. Bollmann, Crystal Defects and Crystalline Interfaces (Springer, Berlin, 1970).

[56] G. A. Prinz, Phys. Rev. Lett. 54, 1051 (1985).

[57] J. R. Cerda, P. L. de Andres, A. Cebollada, R. Miranda, E. Navas, P. Schuster, C. M. Schneider, and J. Kirschner, J. Phys.: Condens. Matter 5, 2055 (1993).

[58] M. Finazzi, L. Duò, and F. Ciccacci, Surf. Sci. Rep. 64, 139 (2009).

[59] Magnetic Properties of Antiferromagnetic Oxide Materials: Surfaces, Interfaces and Thin Films, edited by M. Finazzi, L. Duò, and F. Ciccacci (Wiley, Weinheim, 2010).

[60] M. Riva, A. Picone, G. Bussetti, A. Brambilla, A. Calloni, G. Berti, L. Duò, F. Ciccacci, and M. Finazzi, Surf. Sci. 621, 55 (2014).

[61] A. Calloni, G. Berti, A. Brambilla, M. Riva, A. Picone, G. Bussetti, M. Finazzi, F. Ciccacci, and L. Duò, J. Phys.: Condens. Matter 26, 445001 (2014).

[62] O. O. Brovko, D. I. Bazhanov, H. L. Meyerheim, D. Sander, V. S. Stepanyuk, and J. Kirschner, Surf. Sci. Rep. 69, 159 (2014). 